\title{
Inclusive Human Intention Prediction with Wearable Sensors: Machine Learning Techniques for the Reaching Task Use Case ${ }^{\dagger}$
}

\author{
Leonardo Archetti ${ }^{1}$, Federica Ragni 1,*, Ludovic Saint-Bauzel ${ }^{2}$, Agnès Roby-Brami ${ }^{2}$ and \\ Cinzia Amici 1 \\ 1 Department of Mechanical and Industrial Engineering, University of Brescia, via Branze, 38, 25123 Brescia, \\ Italy; 1.archetti012@studenti.unibs.it (L.A.); cinzia.amici@unibs.it (C.A.) \\ 2 Institut Systèmes Intelligents et de Robotique (ISIR), Sorbonne Université, France \\ ludovic.saint-bauzel@sorbonne-universite.fr(L.S.-B.); roby-brami@isir.upmc.fr (A.R.-B.) \\ * Corresponding author: f.ragni@unibs.it \\ + Presented at the 7th International Electronic Conference on Sensors and Applications, 15-30 November \\ 2020; Available online: https://ecsa-7.sciforum.net/.
}

Published: 15 November 2020

\begin{abstract}
Human intentions prediction is gaining importance with the increase of human-robot interaction challenges in several contexts, like industrial and clinical. This paper compares Linear Discriminant Analysis (LDA) and Random Forest (RF) performance in predicting the intention of moving towards a target during reaching movements, on ten subjects wearing four electromagnetic sensors. LDA and RF prediction accuracy is compared with respect to observation-sample dimension and noise presence, training and prediction time. Both algorithms achieved good accuracy, which improves as the sample dimension increases, although LDA presents better results for the current dataset.
\end{abstract}

Keywords: human intention prediction; wearable sensors; machine learning; reaching movement

\section{Introduction}

Predicting human intentions by collecting and analyzing body signals is one of the main goals in human-robot interaction [1]. Accurate and real-time recognition of human motion intention could allow achieving suitable human-machine coordination [2] for both interactive robotic interfaces, like collaborative robots, and diagnostic systems, such as rehabilitation devices [3].

Several kinds of sensors are currently used to detect body signals, like surface electromyography [2,4], electroencephalography [3], and accelerometers. In recent years, research in human movement pattern recognition with the support of wearable sensors was widely conducted $[2,3,5]$, also considering the effect of the positioning of the sensors in the obtained data [6,7]. Actually, wearable sensors allow noninvasive motion detection, full integration with commercially available devices [1], the possibility to acquire acceleration and velocity to reconstruct the detected movement [8], and the adaptation to inter- and intra-individual variability [9]. Since body signals are strongly affected by repeatability lack [2] and motion is subject-dependent, the challenge in predicting human intention even increases in specific scenarios, like in clinical environment, where the pathological subject can present peculiar motion patterns. In particular, laboratory-based optical motion analysis systems are widely adopted for periodical stroke condition assessment during rehabilitation [10] to obtain multiple bio-signals, useful in recognizing pathological symptoms and to improve the healing rate of rehabilitation [11]. Therefore, the knowledge of expected natural behavior and movement patterns for the healthy subject becomes crucial to perform a correct evaluation. 
Among all the possible movements, the reaching task is fundamental for the activity of daily living [12] because of the relevance of its functional aim.

Since many different strategies can be used to perform the same task [5,13], predictive models and machine learning algorithms are particularly suitable to analyze the signals and predict the movement intention [2]. Developing relevant working methodologies becomes necessary, and machine learning techniques can face the limit of small data amounts. Literature provides various examples of machine learning techniques applied to human motion analysis. For instance, in [14] Linear Discriminant Analysis (LDA), Support Vector Machine and k Nearest Neighbor algorithms have been applied for the identification of natural hand gesture, whereas Li et al. [15] exploited Random Forest algorithm to discriminate 8 different motions of the upper limb.

This study aims to compare LDA and RF machine learning techniques performance in predicting the subject's intention of moving towards a specific direction or target in the illustrative scenario of a reaching movement, using data gathered from wearable electromagnetic sensors.

\section{Materials and Methods}

\subsection{Participants}

A convenience sample of ten healthy subjects (9 right-handed) were recruited from January to October 2009. Inclusion criteria were: (i) age over 18 years old, (ii) no current or previous neurological or orthopedic pathology of the upper arm. The study was approved by CPP Ile de France 8 ethical committee; recruited subjects gave a written informed consent to study participation, and procedures were conducted according to the Declaration of Helsinki.

\subsection{Protocol}

Testing sessions were performed during the morning, in the same environmental conditions. In each session, after a first preliminary trial for procedure familiarization, the operator asked the subject to perform six repetitions of unilateral sitting reaching movement, three with the right arm and three with the left arm. As depicted in [16], each subject was asked to perform the movement 3 times for each combination of direction (internal, middle, external), quote (high, low), and distance (close, far).

The order of the target submitted to the subjects was standardized: close-middle (CM), farinternal (FI), high-external (HE), far-middle (FM), close-external (CE), high-internal (HI), closeinternal (CI), far-external (FE), high-middle (HM). The subjects were required to touch each target with the provided pointer and coming back to the initial condition, moving at a comfortable speed.

\subsection{Experimental Setup}

Subjects were seated on a chair, adjusted so that the table was at the navel level. They wore a wrist splint to which a pointer was rigidly attached to simulate an extended index finger. The subjects' trunk was fixed to the chairback using a wide strap. For each subject, four electromagnetic sensors were placed by a trained operator on i) acromion, ii) upper third of humerus, iii) wrist dorsum and iv) manubrium, respectively. During the acquisitions, the Polhemus Fastrak electromagnetic tracking system was used, which provides the position and orientation of each sensor as timestamped vector triplets $(X, Y, Z)$ and $(\alpha, \beta, \gamma)$, at an output frequency of 30 [Hz] [17]. The system presented a Root Mean Square (RMS) static accuracy is $0.8[\mathrm{~mm}]$ for $X, Y$, and $Z$ receiver position, and $0.15^{\circ}$ for receiver orientation, whereas the resolution is $0.0005\left[\mathrm{~cm} / \mathrm{cm}\right.$ of range] and $0.025^{\circ}$, respectively.

Nine targets were positioned along three directions: (i) middle, on a parasagittal line emanating from the subject's shoulder, (ii) internal, and (iii) external, inclined of $\pm 45^{\circ}$ with respect to the parasagittal line. Targets were composed of red tape of $10[\mathrm{~mm}]$ with a vertical stick of $15[\mathrm{~mm}]$ of diameter. Figure 1 depicts the experimental setup. Distances between targets and subject were parametrized with respect to the anatomical upper limb length, i.e., the distance between the acromion and the end of the pointer. Two distances were considered: (a) far, corresponding to $90 \%$ of the total upper limb length, and (b) close, equal to $65 \%$ of the upper limb length. Six targets were 
placed at $70[\mathrm{~mm}]$ of height from the table level and three were placed above the distal sensor, at the same quote of the acromion from the table surface.

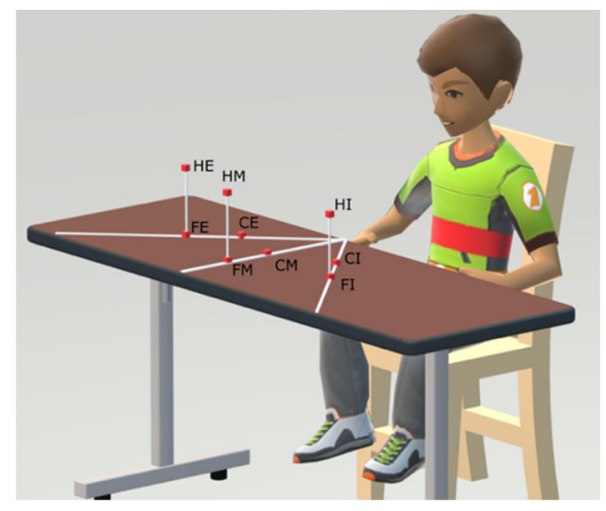

Figure 1. Experimental setup schematization for the initial condition: subject's hand is aligned to the shoulder, placed on a red cross on the table plane, the forearm is mid-prone, the elbow flexed to $90^{\circ}$ and the humerus positioned along the vertical direction.

\subsection{Data Treatment}

Data were elaborated in MATLAB environment using only the information coming from a first sample of the acquired data. To create a dataset of comparable data among trials and among subjects, the acquired signals were trimmed to the actual movement portion. To identify motion starting and ending points, the absolute value of the velocity of the hand has been considered. Therefore, the absolute value of the hand position was calculated for each instant $t$, and the velocity was then computed according to a two-point derivative approximation. According to literature [19,20], the velocity was filtered with a fourth-order zero-phase low-pass Butterworth filter to remove noise, and a cut-off frequency of $3 \mathrm{~Hz}$ has been selected for the filter [20]. The subject resting condition was identified as the mean value of the first and last ten acquired data samples, corresponding to an interval of 0.33 [s]. The starting and ending point of the movement were automatically selected by a custom-made code, as the first and last time instant in which the absolute value of the position first derivative is higher than a selected threshold. This threshold is iteratively identified comparing the variance of the observation sample with the variance that the subject presents at the resting condition: if the variance is higher than $5 \times 10^{-3}[\mathrm{~mm}]$, the threshold values is reduced of $1 \times 10^{-3}[\mathrm{~mm}]$. Acquired data were then normalized in amplitude with respect to subject anthropometric quantities, computed for each patient from hand, arm and shoulder positions.

The relative sensors distances shoulder-to-trunk, arm-to-shoulder and hand-to-arm along the subject resting phase were computed for each trial. For each subject, the average values of these nine quantities were calculated and used as reference values for the data normalization. To simulate data coming from accelerometers placed on subjects, the second derivative of sensors position were computed applying twice the two-point derivative and filtering the result.

Linear and angular position, velocity and acceleration signals were analyzed to identify a set of features for the implementation of the machine learning algorithms, and a portion of the overall motion has been considered as Observation Window (OW). Since the motion duration is unknown in advance, subject- and trial-dependent, two approaches were used to evaluate the OW size: (i) custom window, computing an observation time for each trial using the information on the motion length, and (ii) average window, exploiting all the available data of all the subjects to compute a fixed OW. The evaluated features are the minimum, maximum and root-mean-square of: (i) sensors position (SP) components, (ii) sensors velocity modulus, or first derivative of SP, (iii) sensor acceleration modulus, or the second derivative of SP and iv) Euler angles, respectively. For each trial and subject, the computed features were rescaled to $[-0.80,+0.80]$.

LDA and RF algorithms were implemented and trained using respectively the $85 \%$ and $90 \%$ of the data; in both cases, the data chosen for the training phase were randomly selected. In the testing phase, the remaining $15 \%$ and $10 \%$ of the dataset were used for LDA and RF, respectively. Twenty 
different combinations of these parameters were tested; Table 1 depicts the selected features in the conducted tests. Two different conditions were evaluated for the OW size: 1/10 and 1/7 of the total motion time length. The first ten tests use the custom average approach, whereas the remaining the custom window one. For each test, both the machine learning algorithms were tested. To analyze the algorithms robustness, the same tests were repeated adding Gaussian Noise (noise samples power 0.04 [dBW], load impedance $0.4[\mathrm{ohm}]$ ) to the recorded data before computing the features.

Table 1. The selected features in the conducted tests. In the table, tests with an OW width of $1 / 7$ of the Table 1 . are in regular type.

\begin{tabular}{ccccc}
\hline Tests & $\begin{array}{c}\text { Sensors } \\
\text { Position (SP) }\end{array}$ & $\begin{array}{c}\text { Sensors } \\
\text { Velocity }\end{array}$ & $\begin{array}{c}\text { Sensors } \\
\text { Acceleration }\end{array}$ & $\begin{array}{c}\text { Sensors } \\
\text { Euler Angles }\end{array}$ \\
\hline $1,6,11, \mathbf{1 6}$ & $\mathrm{x}$ & $\mathrm{x}$ & & \\
$2,7,12, \mathbf{1 7}$ & $\mathrm{x}$ & $\mathrm{x}$ & & $\mathrm{x}$ \\
$3, \mathbf{8}, 13, \mathbf{1 8}$ & $\mathrm{x}$ & & & \\
$4,5, \mathbf{9}, \mathbf{1 0}, 14,15, \mathbf{1 9}, \mathbf{2 0}$ & & & $\mathrm{x}$ & \\
\hline
\end{tabular}

LDA and RF algorithms prediction accuracy were computed and compared with respect to data sample dimension, number of considered features, and OW type. Accuracy was computed according to [21,22], and in the case of RF accuracy, the out-of-bag (OOB) approach was also used.

\section{Results}

Table 2 depicts all the obtained results, averaged over 200 consecutive tests. Considering the OW equal to $1 / 10$ of the total movement (average time length of 0.27 [s]), LDA presents in the best case an accuracy in the intention prediction of $86.13 \%$, with a Standard Deviation (SD) of 0.036, and RF an accuracy of $73.73 \%$, with a SD of 0.015 . Increasing the sample at $1 / 7$ of the motion (average time length of 0.37 [s]), the intention prediction accuracy rises at $92.80 \%$ with a SD of 0.027 for LDA, and $84.60 \%$ with a SD of 0.010 for RF. Comparing results obtained from the analysis of data with and without noise, LDA presents a maximum difference equal to $-1.53 \%$ and an average difference of $-0.61 \%$; these values decrease to $-1.28 \%$ and $-0.43 \%$ respectively for RF. Finally, RF algorithm demands an average training time of 1.14 [s] (range: $0.87-1.88$ ), which decreases to an average value of 0.078 [s] (range: 0.035-0.28) for LDA. The prediction time has been computed only for the tests with higher accuracy, i.e., tests $1,2,6$ and 7 . The average prediction time is $31 \times 10^{-4}$ [s] (range $30 \times 10^{-4}-33 \cdot 10^{-4}$ ) for RF and $11 \times 10^{-5}[\mathrm{~s}]\left(\right.$ range $10 \times 10^{-5}-12 \times 10^{-5}$ ) for LDA.

Table 2. Obtained results in RF and LDA algorithms: ori_sgn is the original signal, sgn_N the original signal plus the Gaussian Noise contribute. Results are averaged over 200 consecutive tests.

\begin{tabular}{|c|c|c|c|c|c|c|c|c|c|c|}
\hline \multirow[b]{2}{*}{$\stackrel{\dot{s}}{\stackrel{\infty}{\infty}}$} & \multicolumn{5}{|c|}{ RF } & \multicolumn{5}{|c|}{ LDA } \\
\hline & 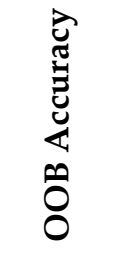 & के & 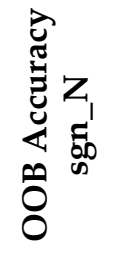 & के & 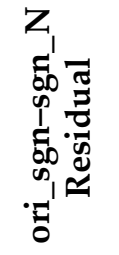 & 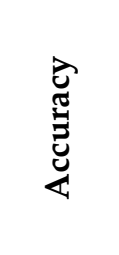 & के & 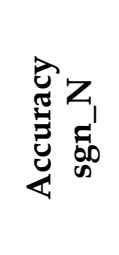 & क & 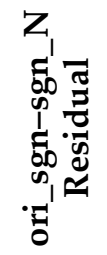 \\
\hline 1 & $72.85 \%$ & 0.032 & $49.40 \%$ & 0.017 & $23.45 \%$ & $81.00 \%$ & 0.042 & $57.04 \%$ & 0.056 & $23.96 \%$ \\
\hline 2 & $73.73 \%$ & 0.015 & $57.48 \%$ & 0.015 & $16.25 \%$ & $86.13 \%$ & 0.036 & $68.73 \%$ & 0.044 & $17.40 \%$ \\
\hline 3 & $61.78 \%$ & 0.016 & $47.91 \%$ & 0.017 & $13.87 \%$ & $73.11 \%$ & 0.045 & $53.12 \%$ & 0.053 & $19.99 \%$ \\
\hline 4 & $59.71 \%$ & 0.015 & $58.71 \%$ & 0.014 & $1.00 \%$ & $58.27 \%$ & 0.054 & $57.40 \%$ & 0.049 & $0.87 \%$ \\
\hline 5 & $71.97 \%$ & 0.013 & $70.69 \%$ & 0.013 & $1.28 \%$ & $73.56 \%$ & 0.047 & $72.81 \%$ & 0.047 & $0.75 \%$ \\
\hline 6 & $82.92 \%$ & 0.011 & $70.10 \%$ & 0.014 & $12.82 \%$ & $88.97 \%$ & 0.032 & $81.07 \%$ & 0.044 & $7.90 \%$ \\
\hline 7 & $84.60 \%$ & 0.01 & $76.38 \%$ & 0.014 & $8.22 \%$ & $92.80 \%$ & 0.027 & $86.60 \%$ & 0.038 & $6.20 \%$ \\
\hline 8 & $76.58 \%$ & 0.013 & $69.69 \%$ & 0.014 & $6.89 \%$ & $85.45 \%$ & 0.041 & $76.36 \%$ & 0.044 & $9.09 \%$ \\
\hline
\end{tabular}




\begin{tabular}{ccccccccccc}
\hline 9 & $64.48 \%$ & 0.016 & $64.34 \%$ & 0.016 & $0.14 \%$ & $63.84 \%$ & 0.054 & $63.26 \%$ & 0.052 & $0.58 \%$ \\
10 & $75.70 \%$ & 0.012 & $75.68 \%$ & 0.011 & $0.02 \%$ & $78.82 \%$ & 0.045 & $78.76 \%$ & 0.044 & $0.06 \%$ \\
11 & $68.04 \%$ & 0.015 & $49.55 \%$ & 0.017 & $18.49 \%$ & $78.80 \%$ & 0.041 & $57.47 \%$ & 0.049 & $21.33 \%$ \\
12 & $71.40 \%$ & 0.015 & $58.21 \%$ & 0.016 & $13.19 \%$ & $83.26 \%$ & 0.042 & $71.17 \%$ & 0.05 & $12.09 \%$ \\
13 & $60.05 \%$ & 0.016 & $50.04 \%$ & 0.017 & $10.01 \%$ & $69.56 \%$ & 0.049 & $54.78 \%$ & 0.045 & $14.78 \%$ \\
14 & $60.92 \%$ & 0.015 & $60.69 \%$ & 0.016 & $0.23 \%$ & $59.57 \%$ & 0.054 & $58.65 \%$ & 0.05 & $0.92 \%$ \\
15 & $72.57 \%$ & 0.013 & $72.47 \%$ & 0.013 & $0.10 \%$ & $74.09 \%$ & 0.048 & $74.31 \%$ & 0.043 & $-0.22 \%$ \\
16 & $80.27 \%$ & 0.012 & $65.22 \%$ & 0.015 & $15.05 \%$ & $86.80 \%$ & 0.04 & $74.97 \%$ & 0.045 & $11.83 \%$ \\
17 & $82.88 \%$ & 0.013 & $74.31 \%$ & 0.013 & $8.57 \%$ & $90.61 \%$ & 0.034 & $84.06 \%$ & 0.041 & $6.55 \%$ \\
18 & $73.65 \%$ & 0.012 & $66.25 \%$ & 0.014 & $7.40 \%$ & $81.15 \%$ & 0.044 & $73.81 \%$ & 0.047 & $7.34 \%$ \\
19 & $66.26 \%$ & 0.014 & $65.80 \%$ & 0.015 & $0.46 \%$ & $70.04 \%$ & 0.048 & $69.62 \%$ & 0.045 & $0.42 \%$ \\
20 & $78.17 \%$ & 0.011 & $77.99 \%$ & 0.011 & $0.18 \%$ & $83.59 \%$ & 0.04 & $82.06 \%$ & 0.044 & $1.53 \%$ \\
\hline
\end{tabular}

\section{Discussion}

Comparing the intention prediction performance of the algorithms with respect to the OW size, better results were obtained when larger windows were considered. Nevertheless, a reasonable limit should be imposed to the window size to avoid that the intention is predicted when the movement is close to its end. For features regarding Euler angles, sensor position and speed, as the window width increases from $1 / 10$ to $1 / 7$, accuracy improves by more than $10 \%$ for both algorithms, in all the tests. When acceleration features are considered, the improvement achieved by a wider OW is about 5 percentage points, with a SD close to $1 \%$. This behavior can be likely explained considering that the features calculated on the acceleration are subjected to the noise generated by the double derivation. Nevertheless, it represents a qualitative estimation of the results that the algorithms could provide, processing acceleration data from an accelerometer or inertial measurement units (IMU). This interpretation is supported by the results of the noise-added data: in those tests where the acceleration features are considered, noise does not affect significantly the accuracy. This could be justified by the fact that the computed acceleration signal is already noisy. Focusing on the time dimension, LDA reveals considerably shorter training times than RF. To decrease RF training time, the number of trees in the forest can be reduced: a preliminary analysis revealed that after about 40 trees, the accuracy of the algorithm tends to a horizontal asymptote. In the same way, LDA presents significantly shorter prediction times than RF in all the tests.

\section{Conclusions}

This paper investigates the human reaching movement comparing the performance of LDA and $\mathrm{RF}$ to predict subjects' intention of moving towards a specific direction or target, when analyzing data gathered from wearable electromagnetic sensors. A campaign on ten healthy subjects was performed, and features on measured and computed signals were evaluated. The analyses revealed that the OW size is a crucial quantity: the wider the window, the better the prediction performance. The introduction of noise does not significantly affect the prediction performance of both the algorithms when acceleration features are also considered. For both machine learning techniques a good accuracy is demonstrated, although LDA presents more promising results in terms of accuracy, training time and prediction time with the current dataset.

Further experimental campaigns, including different kinds of sensors or their positioning strategies, are currently under evaluation. In fact, actual acceleration data gathered from accelerometers and/or IMU inertial sensors would allow an experimental validation of the hypotheses about the acceleration features. Besides, the employment of different wearable sensors could make the acquisition system less invasive for the subject and more flexible, promoting for instance the use of widely spread and cheaper electronic devices, like smartphones or smartwatches.

Author Contributions: Conceptualization, L.A., F.R., L.S.-B., A.R.-B. and C.A.; methodology, L.S.-B. and C.A.; software, L.A. and F.R.; formal analysis, L.S.-B., A.R.-B. and C.A.; investigation, L.S.-B. and A.R.-B.; data curation, L.A., F.R. and C.A.; writing-original draft preparation, L.A., F.R. and C.A.; writing-review and editing, L.S.-B. and A.R.-B. All authors have read and agreed to the published version of the manuscript. 
Acknowledgments: The authors warmly thank Johanna Robertson for the support in data collection process.

Conflicts of Interest: The authors declare no conflict of interest.

\section{References}

1. Preatoni, E.; Nodari, S.; Lopomo, N.F. Supervised Machine Learning Applied to Wearable Sensor Data Can Accurately Classify Functional Fitness Exercises Within a Continuous Workout. Front. Bioeng. Biotechnol. 2020, 8, 1-13.

2. Zhang, L.; Liu, G.; Han, B.; Wang, Z.; Zhang, T. SEMG Based Human Motion Intention Recognition. J. Robot. 2019, 2019, 3679174.

3. Cangelosi, A.; Invitto, S. Human-Robot Interaction and Neuroprosthetics: A review of new technologies. IEEE Consum. Electron. Mag. 2017, 6, 24-33.

4. Balasubramanian, S.; Garcia-Cossio, E.; Birbaumer, N.; Burdet, E.; Ramos-Murguialday, A. Is EMG a Viable Alternative to BCI for Detecting Movement Intention in Severe Stroke? IEEE Trans. Biomed. Eng. 2018, 65, 2790-2797.

5. Merad, M.; de Montalivet, É.; Touillet, A.; Martinet, N.; Roby-Brami, A.; Jarrassé, N. Can we achieve intuitive prosthetic elbow control based on healthy upper limb motor strategies? Front. Neurorobot. 2018, $12,1$.

6. Ragni, F.; Amici, C.; Borboni, A.; Faglia, R.; Cappellini, V.; Pedersini, P.; Villafañe, J.H. Effects of Soft Tissue Artifact in the Measurement of Hand Kinematics. Int. Rev. Mech. Eng. 2020, 14, 230-242.

7. Negrini, S.; Serpelloni, M.; Amici, C.; Gobbo, M.; Silvestro, C.; Buraschi, R.; Borboni, A.; Crovato, D.; Lopomo, N.F. Use of wearable inertial sensor in the assessment of Timed-Up-and-Go Test: Influence of device placement on temporal variable estimation. In Lecture Notes of the Institute for Computer Sciences, Social-Informatics and Telecommunications Engineering, Proceedings of the International Conference on Wireless Mobile Communication and Healthcare, Milan, Italy, 14-16 November 2016; Springer: Cham, Switzerland, 2017; Volume 192.

8. Saint-Bauzel, L.; Pasqui, V.; Morel, G.; Gas, B. Real-time human posture observation from a small number of joint measurements. In Proceedings of the 2007 IEEE/RSJ International Conference on Intelligent Robots and Systems, San Diego, CA, USA, 29 October-2 November 2007; pp. 3956-3961.

9. Endres, F.; Hess, J.; Burgard, W. Graph-based action models for human motion classification. In Proceedings of the ROBOTIK 2012, 7th German Conference on Robotics, Munich, Germany, 21-22 May 2012; pp. 1-6.

10. Yang, C.; Kerr, A.; Stankovic, V.; Stankovic, L.; Rowe, P. Upper limb movement analysis via marker tracking with a single-camera system. In Proceedings of the 2014 IEEE International Conference on Image Processing (ICIP), Paris, France, 27-30 October 2014; pp. 2285-2289.

11. He, J.; Chen, S.; Guo, Z.; Pirbhulal, S.; Wu, W.; Feng, J.; Dan, G. A comparative study of motion recognition methods for efficacy assessment of upper limb function. Int. J. Adapt. Control Signal Process. 2018, 33, 12481256.

12. Roby-Brami, A.; Feydy, A.; Combeaud, M.; Biryukova, E.V.; Bussel, B.; Levin, M.F. Motor compensation and recovery for reaching in stroke patients. Acta Neurol. Scand. 2003, 107, 369-381.

13. Molteni, F.; Gasperini, G.; Cannaviello, G.; Guanziroli, E. Exoskeleton and End-Effector Robots for Upper and Lower Limbs Rehabilitation: Narrative Review. PM R 2018, 10, S174-S188.

14. Romaszewski, M.; Glomb, P.; Gawron, P. Natural hand gestures for human identification in a HumanComputer Interface. In Proceedings of the 2014 4th International Conference on Image Processing Theory, Tools and Applications (IPTA), Paris, France, 14-17 October 2014.

15. Li, B.; Bai, B.; Han, C. Upper body motion recognition based on key frame and random forest regression. Multimed. Tools Appl. 2020, 79, 5197-5212.

16. Robertson, J.V.G.; Roche, N.; Roby-Brami, A. Influence of the side of brain damage on postural upper-limb control including the scapula in stroke patients. Exp. Brain Res. 2012, 218, 141-155.

17. Polhemus. SPACE FASTRAK User's Manuel; Revision F.; 1993.

18. Amici, C.; Ghidoni, M.; Ceresoli, F.; Gaffurini, P.; Bissolotti, L.; Mor, M.; Fausti, D.; Anton, M.; Ragni, F.; Tiboni, M. Preliminary Validation of a Device for the Upper and Lower Limb Robotic Rehabilitation. In Proceedings of the ICMT 2019|23rd International Conference on Mechatronics Technology, Salerno, Italy, 23-26 October 2019. 
19. Godfrey, A.; Bourke, A.K.; Ólaighin, G.M.; van de Ven, P.; Nelson, J. Activity classification using a single chest mounted tri-axial accelerometer. Med. Eng. Phys. 2011, 33, 1127-1135.

20. Moyle, W.; Arnautovska, U.; Ownsworth, T.; Jones, C. Potential of telepresence robots to enhance social connectedness in older adults with dementia: An integrative review of feasibility. Int. Psychogeriatr. 2017, 29, 1951-1964.

21. Nuzzi, C.; Pasinetti, S.; Lancini, M. Deep Learning-Based Hand Collaborative Robots. IEEE Instrum. Meas. Mag. 2019, 22, 44-51.

22. Nuzzi, C.; Pasinetti, S.; Lancini, M.; Docchio, F.; Sansoni, G. Deep Learning Based Machine Vision: First Steps Towards a Hand Gesture Recognition Set Up for Collaborative Robots. In Proceedings of the 2018 2018 Workshop on Metrology for Industry 4.0 and IoT, Brescia, Italy, 16-18 April 2018; pp. 28-33.

Publisher's Note: MDPI stays neutral with regard to jurisdictional claims in published maps and institutional affiliations.

(C) 2020 by the authors. Submitted for possible open access publication under the terms and conditions of the Creative Commons Attribution (CC BY) license (http://creativecommons.org/licenses/by/4.0/). 